\title{
Prospective urban and rural epidemiology Poland - study design
}

\author{
Katarzyna Zatońska', Witold A. Zatoński' ${ }^{2,3}$, Andrzej Szuba ${ }^{4,5}$ \\ 'Department of Social Medicine, Wroclaw Medical University, Wroclaw, Poland \\ ${ }^{2}$ Health Promotion Foundation, Nadarzyn, Poland \\ ${ }^{3}$ Higher Vocational State School in Kalisz, Poland \\ ${ }^{4}$ Division of Angiology, Wroclaw Medical University, Wroclaw, Poland \\ ${ }^{5}$ Department of Internal Medicine, $4^{\text {th }}$ Military Hospital in Wroclaw, Poland
}

ADDRESS FOR CORRESPONDENCE: Prof. Witold A. Zatoński, Health Promotion Foundation, 51 Mszczonowska Street, 05-830 Nadarzyn, Poland, e-mail: wazatonski@promocjazdrowia.pl

\section{INTRODUCTION}

Health improvements marked with impressive increase of life expectancy can be seen throughout human populations. The first half of the twentieth century brought a dramatic decline in childhood and infectious diseases, but this was accompanied with a growing prevalence of chronic conditions. On average the population gained 20 years of life between 1948 and 1998, when life expectancy at birth reached 66 years. Socio-economic development and new consumption patterns are the main driving forces of this new health and life experience. Economic growth takes place in cycles with the most turbulent processes in low- and middle-income countries. Urbanisation is one of most visible results of this change. The prognosis of this situation is that by 2020 over $85 \%$ of the world's population will live in developing countries. It brings an urgent need to understand the connections between the forces and processes behind socioeconomic development and the burden of the diseases especially chronic diseases, like obesity, diabetes, and cardiovascular diseases, accompanying this process. The PURE study is a research project attempting to challenge issues. It is a global research project established to elaborate a strategy to link and analyse environmental (cultural-socio-economic) factors with individual biological (metabolic, genetic, and lifestyle) conditions. Participants were recruited from 21 low-, middle-, and high-income countries, such as: Argentina, Brazil, Chile, China, India, Tanzania, Thailand, Turkey, Zimbabwe, and the United Arab Emirates, representing different parts of the world, reaching in total over 150,000 people. The research centre is located in the Population Health Research Institute in Hamilton, Can- ada. The principal investigator of the project is Professor Salim Yusuf $[1,2]$.

The average life expectancy in Poland is growing every year. In 2015 it was 73.6 years for males and 81.6 for females. This difference between genders is present in urban and rural areas (urban: $\mathrm{M}-74, \mathrm{~F}-81.5$, rural: $\mathrm{M}$ - 73, F - 81.7). Unfortunately, we are still behind in comparison to western European countries, which is a reason to proceed with studies concerning medical issues of Poles and ways to solve them despite the increase in life expectancy during the last 25 years. Answers to the mentioned issues might be provided by the PURE study.

\section{RESEARCH HYPOTHESIS}

Risk factors arising from environmental, genetic, and psychosocial background lead to increases in the incidence of noncommunicable diseases (NCDs).

The project aim is to describe this processes and develop actions targeting major health challenges like the increase in obesity, diabetes, and cardiovascular disease as well as other noncommunicable disease prevalence.

\section{GENERAL AIMS OF THE STUDY}

1. Assessment of social change impact on lifestyle and health risk factors exposure.

2. Assessment of the impact of health risk factors on the development of obesity, diabetes, and cardiovascular morbidities.

3. Assessment of the impact of individual health risk factors together with socio-economic factors (urbanisation, increase in mechanisation, health care delivery) on the modification of noncommunicable disease risk factors. 


\section{GENERAL CHARACTERISTICS OF THE PROJECT}

In Poland the project started back in 2007 at Wroclaw Medical University. Data is collected cyclically with threeyear intervolution; the research is planned for 12 years (2007-2019). At present nine-year follow-up has started.

Overall 2036 people aged between 30 and 85 years were enrolled to the study by the year 2010 . The project aims to examine households enrolled to the study and their communities; 1210 people with urban residence and 826 people with rural residence. The urban centre was organised around the Medical University, and the rural centre was in Węgry - a small village in the General Practice of Doctor Ireneusz Zatoński.

At the first stage of the research the participants were interviewed (face to face) with six study questionnaire forms.

The six questionnaires:

1. Household questionnaire - household level.

2. Family questionnaire - household level.

3. Health questionnaire - individual level.

4. International Physical Activity Questionnaire - short form - IPAQ - individual level.

5. Food Frequency Questionnaire FFQ - individual level.

6. Last 24-hours food intake questionnaire - individual level.

\section{DESCRIPTIONS OF QUESTIONNAIRE}

Household questionnaire (10 questions) concerned household living and socio-economic conditions.

Family questionnaire (4 questions) focused on basic family members' information, thus not including participation in the research due to lack of consent to participate in the project or age.

Health questionnaire (42 questions) concerned medical history, in particular chronic conditions with social gradient (ischaemic heart disease, hypertension, cancer, respiratory diseases), as well as disabilities, injuries, depression, social exclusion, and family background. It also included questions on basic health risk factors, such as smoking tobacco products and alcohol consumption.

International Physical Activity Questionnaire (17 questions) was used to assess the physical activity of every respondent at different locations: during working hours, at home, and leisure activities. It categorised people into three groups with respect to the level of physical activity intense, average, and low.

Food Frequency Questionnaire (173 questions) recorded the consumption amounts of specific food products during a period of one year - the questionnaire was validated [3].

Last 24-hours food intake questionnaire recorded consumption of food products during last 24 hours prior to the questionnaire.

Basic anthropometric data (height and body mass to calculate BMI; hip and waist circumference to calculate WHR), blood pressure, spirometry test, ECG test, and biological material (blood, urine) was collected from the participants.

During the study all patients after 3,6, 9, and 12 years of project cycle are invited for medical check-up; besides this, telephone health survey on hospital admissions and new diagnosis by contact is carried out every year.

\section{KEY FINDINGS}

Selected characteristic distribution of participants is listed in Table 1. Mean age at baseline was 54 years. The majority of our PURE cohort individuals were married or living in co-habiting relationships (74.3\%), and there was a higher proportion of men than women. The majority of the cohort members were employed at baseline (55.7\%). In the youngest, 30-44-year age group, the proportion of full-time employed was higher $(92.9 \%$ in men and $85.3 \%$ in women).

Most of the cohort members completed secondary (39.3\%) and higher education (29.8\%). More women than men completed only secondary education $(41.8 \%$ of women vs. $35.1 \%$ of men); on the other hand, the proportions between sexes were almost the same in the case of higher education ( $31.7 \%$ of men vs. $28.7 \%$ of women).

Over $40 \%$ of the cohort members were retired $(60.3 \%$ of unemployed were retired because of advanced age vs. $23.6 \%$ as a result of disease). $59.4 \%$ of cohort members lived in the urban area and $40.6 \%$ lived in the rural area; these proportions were similar across age groups and sexes (with the exception of older women: $47.7 \%$ lived in the urban and $52.3 \%$ in the rural area).

One-fifth $(21.0 \%)$ of the cohort members were current smokers. The prevalence of current smokers was higher among men than women, with the highest prevalence in the 45-64-year age group of men. Almost half of the population were never smokers $(47.3 \%)$; more women than men were never smokers $(54.7 \%$ of women vs. $34.8 \%$ of men). The majority of the cohort members reported that alcohol products were used currently by them $(67.3 \%) ; 10.3 \%$ of the population formerly used alcohol products and $22.4 \%$ never used them. Surprisingly, more women than men reported current use of alcohol ( $61.5 \%$ of women vs. $11.6 \%$ of men). Among men, current alcohol use was more prevalent in the 30-44-year age group, whereas among women it was more prevalent in the 45-64-year age group. Mean body mass index (BMI) was $28.1 \mathrm{~kg} / \mathrm{m}^{2}$ for the entire cohort. Normal-range BMI was highest in younger women $(55.8 \%)$ and lowest in older men (17.3\%). $40.1 \%$ of the cohort was overweight and $31.1 \%$ were obese according to BMI. Obesity was more prevalent among older women, but overweight was more prevalent among older men [4-7].

Only half of the surveyed (48.2\%) declared lack of chronic conditions (Table 2). Rural living conditions appeared to be statistically significant $(\mathrm{OR}=1.9496$; CI 1.296-1.6124). The risk is independent of sex (OR = 0.9134; CI 0.7627-1.0938). Highest risk factor was noted 
TABLE 1. Basic characteristics of participants: PURE Poland study

\begin{tabular}{|c|c|c|c|c|c|c|c|c|c|c|}
\hline Characteristics & Missing & $\begin{array}{l}\text { Valid } \\
N(\%)\end{array}$ & $\begin{array}{l}\text { Men } \\
\text { aged } \\
30-44\end{array}$ & $\begin{array}{l}\text { Men } \\
\text { aged } \\
45-64\end{array}$ & $\begin{array}{l}\text { Men } \\
\text { aged } \\
>64\end{array}$ & $\begin{array}{l}\text { Men } \\
\text { total }\end{array}$ & $\begin{array}{l}\text { Women } \\
\text { aged } \\
30-44\end{array}$ & $\begin{array}{c}\text { Women } \\
\text { aged } \\
45-64\end{array}$ & $\begin{array}{c}\text { Women } \\
\text { aged } \\
>64\end{array}$ & $\begin{array}{l}\text { Women } \\
\text { total }\end{array}$ \\
\hline Demographics & & $\begin{array}{l}2036 \\
(100)\end{array}$ & $\begin{array}{l}159 \\
(7.8)\end{array}$ & $\begin{array}{c}468 \\
(23.0)\end{array}$ & $\begin{array}{l}127 \\
(6.2)\end{array}$ & $\begin{array}{c}754 \\
(37.0)\end{array}$ & $\begin{array}{l}199 \\
(9.8)\end{array}$ & $\begin{array}{c}884 \\
(43.4)\end{array}$ & $\begin{array}{l}199 \\
(9.8)\end{array}$ & $\begin{array}{l}1282 \\
(63.0)\end{array}$ \\
\hline $\begin{array}{l}\text { Age at baseline } \\
\text { (mean) }\end{array}$ & & $\begin{array}{c}54 \\
( \pm 10)\end{array}$ & $\begin{array}{c}40 \\
( \pm 3.1)\end{array}$ & $\begin{array}{c}55 \\
( \pm 5)\end{array}$ & $\begin{array}{c}68 \\
( \pm 3)\end{array}$ & $\begin{array}{c}54 \\
( \pm 10.0)\end{array}$ & $\begin{array}{c}39 \\
( \pm 3.3)\end{array}$ & $\begin{array}{c}55 \\
( \pm 5)\end{array}$ & $\begin{array}{c}69 \\
( \pm 4)\end{array}$ & $\begin{array}{c}55 \\
( \pm 9.7)\end{array}$ \\
\hline Material status & 6 & & & & & 4 & & & & 2 \\
\hline $\begin{array}{l}\text { Married/living } \\
\text { together }\end{array}$ & & $\begin{array}{l}1508 \\
(74.3)\end{array}$ & $\begin{array}{c}126 \\
(80.8)\end{array}$ & $\begin{array}{c}408 \\
(87.4)\end{array}$ & $\begin{array}{c}111 \\
(87.4)\end{array}$ & $\begin{array}{c}654 \\
(86.0)\end{array}$ & $\begin{array}{c}155 \\
(77.9)\end{array}$ & $\begin{array}{c}621 \\
(70.4)\end{array}$ & $\begin{array}{c}87 \\
(43.7)\end{array}$ & $\begin{array}{c}863 \\
(67.4)\end{array}$ \\
\hline $\begin{array}{l}\text { Separated/di- } \\
\text { vorced/widowed }\end{array}$ & & $\begin{array}{c}376 \\
(18.5)\end{array}$ & $\begin{array}{c}8 \\
(5.1) \\
\end{array}$ & $\begin{array}{c}33 \\
(7.0)\end{array}$ & $\begin{array}{c}11 \\
(8.7)\end{array}$ & $\begin{array}{c}52 \\
(6.9)\end{array}$ & $\begin{array}{c}24 \\
(12.1)\end{array}$ & $\begin{array}{c}197 \\
(22.3)\end{array}$ & $\begin{array}{c}103 \\
(51.8)\end{array}$ & $\begin{array}{c}324 \\
(25.3)\end{array}$ \\
\hline $\begin{array}{l}\text { Newer married/ } \\
\text { single }\end{array}$ & & $\begin{array}{l}146 \\
(7.2)\end{array}$ & $\begin{array}{c}22 \\
(14.1)\end{array}$ & $\begin{array}{c}26 \\
(5.6)\end{array}$ & $\begin{array}{c}5 \\
(3.9)\end{array}$ & $\begin{array}{c}53 \\
(7.1)\end{array}$ & $\begin{array}{c}20 \\
(10.0)\end{array}$ & $\begin{array}{c}64 \\
(7.3) \\
\end{array}$ & $\begin{array}{c}9 \\
(4.5)\end{array}$ & $\begin{array}{c}93 \\
(7.3) \\
\end{array}$ \\
\hline Education & 11 & & & & & 5 & & & & 6 \\
\hline Primary & & $\begin{array}{c}302 \\
(14.9)\end{array}$ & $\begin{array}{c}12 \\
(7.7) \\
\end{array}$ & $\begin{array}{c}51 \\
(10.9)\end{array}$ & $\begin{array}{c}39 \\
(31.0)\end{array}$ & $\begin{array}{c}102 \\
(13.6)\end{array}$ & $\begin{array}{c}11 \\
(5.5)\end{array}$ & $\begin{array}{c}109 \\
(12.4)\end{array}$ & $\begin{array}{c}80 \\
(40.8) \\
\end{array}$ & $\begin{array}{c}200 \\
(15.6) \\
\end{array}$ \\
\hline Vocational & & $\begin{array}{c}324 \\
(16.0)\end{array}$ & $\begin{array}{c}32 \\
(20.7)\end{array}$ & $\begin{array}{c}103 \\
(22.0)\end{array}$ & $\begin{array}{c}12 \\
(9.5)\end{array}$ & $\begin{array}{c}147 \\
(19.6)\end{array}$ & $\begin{array}{c}35 \\
(17.6)\end{array}$ & $\begin{array}{c}129 \\
(14.6)\end{array}$ & $\begin{array}{c}13 \\
(6.6)\end{array}$ & $\begin{array}{c}177 \\
(13.9)\end{array}$ \\
\hline Secondary & & $\begin{array}{c}796 \\
(39.3) \\
\end{array}$ & $\begin{array}{c}43 \\
(27.7) \\
\end{array}$ & $\begin{array}{c}186 \\
(39.7) \\
\end{array}$ & $\begin{array}{c}34 \\
(27.0) \\
\end{array}$ & $\begin{array}{c}263 \\
(35.1) \\
\end{array}$ & $\begin{array}{c}65 \\
(32.7) \\
\end{array}$ & $\begin{array}{c}401 \\
(45.5) \\
\end{array}$ & $\begin{array}{c}67 \\
(34.2) \\
\end{array}$ & $\begin{array}{c}533 \\
(41.8) \\
\end{array}$ \\
\hline Higher & & $\begin{array}{c}603 \\
(29.8)\end{array}$ & $\begin{array}{c}68 \\
(43.9)\end{array}$ & $\begin{array}{c}128 \\
(27.4)\end{array}$ & $\begin{array}{c}41 \\
(32.5)\end{array}$ & $\begin{array}{c}237 \\
(31.7)\end{array}$ & $\begin{array}{c}88 \\
(44.2)\end{array}$ & $\begin{array}{c}242 \\
(27.5)\end{array}$ & $\begin{array}{c}36 \\
(18.4)\end{array}$ & $\begin{array}{c}366 \\
(28.7)\end{array}$ \\
\hline $\begin{array}{l}\text { Employment } \\
\text { status }\end{array}$ & 32 & & & & & 3 & & & & 29 \\
\hline Employed & & $\begin{array}{l}1117 \\
(55.7)\end{array}$ & $\begin{array}{c}142 \\
(92.9)\end{array}$ & $\begin{array}{c}323 \\
(69.0)\end{array}$ & $\begin{array}{c}21 \\
(16.5)\end{array}$ & $\begin{array}{c}486 \\
(64.7)\end{array}$ & $\begin{array}{c}168 \\
(85.3)\end{array}$ & $\begin{array}{c}453 \\
(52.6)\end{array}$ & $\begin{array}{c}10 \\
(5.2)\end{array}$ & $\begin{array}{l}1631 \\
(50.4)\end{array}$ \\
\hline Unemployed & & $\begin{array}{c}887 \\
(44.3) \\
\end{array}$ & $\begin{array}{c}14 \\
(7.1)\end{array}$ & $\begin{array}{c}145 \\
(31.0) \\
\end{array}$ & $\begin{array}{c}106 \\
(83.5) \\
\end{array}$ & $\begin{array}{c}265 \\
(35.3) \\
\end{array}$ & $\begin{array}{c}29 \\
(14.7) \\
\end{array}$ & $\begin{array}{c}409 \\
(47.4) \\
\end{array}$ & $\begin{array}{c}184 \\
(94.8) \\
\end{array}$ & $\begin{array}{c}622 \\
(49.6) \\
\end{array}$ \\
\hline $\begin{array}{l}\text { Cessation } \\
\text { of employ- } \\
\text { ment, retired } \\
\text { because of age }\end{array}$ & & $\begin{array}{c}535 \\
60.3 \\
\% \text { overall } \\
\text { unem- } \\
\text { ployed }\end{array}$ & & $\begin{array}{c}60 \\
(41.4)\end{array}$ & $\begin{array}{c}82 \\
(77.4)\end{array}$ & $\begin{array}{c}142 \\
(53.6)\end{array}$ & & $\begin{array}{c}254 \\
(62.1)\end{array}$ & $\begin{array}{c}139 \\
(75.0)\end{array}$ & $\begin{array}{c}393 \\
(63.2)\end{array}$ \\
\hline $\begin{array}{l}\text { Cessation } \\
\text { of employment, } \\
\text { retired because } \\
\text { of illness }\end{array}$ & & $\begin{array}{c}209 \\
23.6 \\
\% \text { overall } \\
\text { unem- } \\
\text { ployed }\end{array}$ & $\begin{array}{c}4 \\
(28.8)\end{array}$ & $\begin{array}{c}40 \\
(27.6)\end{array}$ & $\begin{array}{c}24 \\
(22.6)\end{array}$ & $\begin{array}{c}68 \\
(25 .) 7\end{array}$ & $\begin{array}{c}4 \\
(13.8)\end{array}$ & $\begin{array}{c}91 \\
(22.3)\end{array}$ & $\begin{array}{c}46 \\
(25.0)\end{array}$ & $\begin{array}{c}141 \\
(22.7)\end{array}$ \\
\hline \multicolumn{11}{|l|}{$\begin{array}{l}\text { Place } \\
\text { of residence }\end{array}$} \\
\hline Urban & & $\begin{array}{r}1210 \\
(59.4) \\
\end{array}$ & $\begin{array}{c}96 \\
(60.4) \\
\end{array}$ & $\begin{array}{c}287 \\
(61.3) \\
\end{array}$ & $\begin{array}{c}76 \\
(59.8) \\
\end{array}$ & $\begin{array}{c}459 \\
(60.9) \\
\end{array}$ & $\begin{array}{c}107 \\
(53.8) \\
\end{array}$ & $\begin{array}{c}549 \\
(62.1) \\
\end{array}$ & $\begin{array}{c}95 \\
(47.7) \\
\end{array}$ & $\begin{array}{c}751 \\
(58.6) \\
\end{array}$ \\
\hline Rural & & $\begin{array}{c}826 \\
(40.6)\end{array}$ & $\begin{array}{c}63 \\
(39.6)\end{array}$ & $\begin{array}{c}181 \\
(38.7)\end{array}$ & $\begin{array}{c}51 \\
(40.2)\end{array}$ & $\begin{array}{c}295 \\
(39.1)\end{array}$ & $\begin{array}{c}92 \\
(46.2)\end{array}$ & $\begin{array}{c}335 \\
(37.9)\end{array}$ & $\begin{array}{c}104 \\
(52.3)\end{array}$ & $\begin{array}{c}531 \\
(41.4)\end{array}$ \\
\hline \multicolumn{11}{|l|}{ Lifestyle profile } \\
\hline Smoking & 6 & & & & & 3 & & & & 3 \\
\hline $\begin{array}{l}\text { Current } \\
\text { smokers }\end{array}$ & & $\begin{array}{c}425 \\
(21.0)\end{array}$ & $\begin{array}{c}39 \\
(25.0)\end{array}$ & $\begin{array}{c}127 \\
(27.1)\end{array}$ & $\begin{array}{c}14 \\
(11.0)\end{array}$ & $\begin{array}{c}180 \\
(24.0)\end{array}$ & $\begin{array}{c}47 \\
(23.6)\end{array}$ & $\begin{array}{c}190 \\
(21.6)\end{array}$ & $\begin{array}{c}8 \\
(4.0)\end{array}$ & $\begin{array}{c}254 \\
(19.2)\end{array}$ \\
\hline
\end{tabular}


TABLE 1. Cont.

\begin{tabular}{|c|c|c|c|c|c|c|c|c|c|c|}
\hline Characteristics & Missing & $\begin{array}{l}\text { Valid } \\
N(\%)\end{array}$ & $\begin{array}{l}\text { Men } \\
\text { aged } \\
30-44\end{array}$ & $\begin{array}{l}\text { Men } \\
\text { aged } \\
45-64\end{array}$ & $\begin{array}{l}\text { Men } \\
\text { aged } \\
>64\end{array}$ & $\begin{array}{l}\text { Men } \\
\text { total }\end{array}$ & $\begin{array}{c}\text { Women } \\
\text { aged } \\
30-44\end{array}$ & $\begin{array}{c}\text { Women } \\
\text { aged } \\
45-64\end{array}$ & $\begin{array}{c}\text { Women } \\
\text { aged } \\
>64\end{array}$ & $\begin{array}{c}\text { Women } \\
\text { total }\end{array}$ \\
\hline Ex-smokers & & $\begin{array}{c}644 \\
(31.7)\end{array}$ & $\begin{array}{c}40 \\
(25.6)\end{array}$ & $\begin{array}{c}203 \\
(43.4)\end{array}$ & $\begin{array}{c}67 \\
(52.8)\end{array}$ & $\begin{array}{c}310 \\
(41.3)\end{array}$ & $\begin{array}{c}44 \\
(22.1)\end{array}$ & $\begin{array}{c}251 \\
(28.5)\end{array}$ & $\begin{array}{c}39 \\
(19.6)\end{array}$ & $\begin{array}{c}334 \\
(26.1)\end{array}$ \\
\hline $\begin{array}{l}\text { Never } \\
\text { smokers }\end{array}$ & & $\begin{array}{c}961 \\
(47.3)\end{array}$ & $\begin{array}{c}77 \\
(49.4)\end{array}$ & $\begin{array}{c}138 \\
(29.5)\end{array}$ & $\begin{array}{c}46 \\
(36.2)\end{array}$ & $\begin{array}{c}261 \\
(34.8)\end{array}$ & $\begin{array}{c}108 \\
(54.3)\end{array}$ & $\begin{array}{c}440 \\
(49.9)\end{array}$ & $\begin{array}{c}152 \\
(76.4)\end{array}$ & $\begin{array}{c}700 \\
(54.7)\end{array}$ \\
\hline Alcohol & 6 & & & & & 3 & & & & 3 \\
\hline $\begin{array}{l}\text { Currently } \\
\text { use alcohol } \\
\text { products }\end{array}$ & & $\begin{array}{l}1367 \\
(67.3)\end{array}$ & $\begin{array}{c}134 \\
(85.8)\end{array}$ & $\begin{array}{c}370 \\
(79.1)\end{array}$ & $\begin{array}{c}76 \\
(59.8)\end{array}$ & $\begin{array}{c}87 \\
(11.6)\end{array}$ & $\begin{array}{c}127 \\
(63.8)\end{array}$ & $\begin{array}{c}577 \\
(65.5)\end{array}$ & $\begin{array}{c}83 \\
(41.7)\end{array}$ & $\begin{array}{c}787 \\
(61.5)\end{array}$ \\
\hline $\begin{array}{l}\text { Formerly } \\
\text { used alcohol } \\
\text { products }\end{array}$ & & $\begin{array}{c}208 \\
(10.3)\end{array}$ & $\begin{array}{c}11 \\
(7.1)\end{array}$ & $\begin{array}{c}48 \\
(10.2)\end{array}$ & $\begin{array}{c}28 \\
(22.1)\end{array}$ & $\begin{array}{c}580 \\
(77.2)\end{array}$ & $\begin{array}{c}18 \\
(9.1)\end{array}$ & $\begin{array}{c}68 \\
(7.7)\end{array}$ & $\begin{array}{c}35 \\
(17.6)\end{array}$ & $\begin{array}{l}121 \\
(9.5)\end{array}$ \\
\hline $\begin{array}{l}\text { Never used } \\
\text { alcohol } \\
\text { products }\end{array}$ & & $\begin{array}{c}455 \\
(22.4)\end{array}$ & $\begin{array}{c}11 \\
(7.1)\end{array}$ & $\begin{array}{c}50 \\
(10.7)\end{array}$ & $\begin{array}{c}23 \\
(18.1)\end{array}$ & $\begin{array}{c}84 \\
(11.2)\end{array}$ & $\begin{array}{c}54 \\
(27.1)\end{array}$ & $\begin{array}{c}236 \\
(26.8)\end{array}$ & $\begin{array}{c}81 \\
(40.7)\end{array}$ & $\begin{array}{c}371 \\
(29.0)\end{array}$ \\
\hline $\begin{array}{l}\text { Body mass index } \\
\text { (mean) }\end{array}$ & 1 & $\begin{array}{c}28.1 \\
( \pm 5.1)\end{array}$ & $\begin{array}{c}27.7 \\
( \pm 4.3) \\
\end{array}$ & $\begin{array}{c}28.9 \\
( \pm 4.7)\end{array}$ & $\begin{array}{r}28.2 \\
( \pm 3.5) \\
\end{array}$ & $\begin{array}{c}28.5 \\
( \pm 4.5)\end{array}$ & $\begin{array}{c}25.1 \\
( \pm 4.5)\end{array}$ & $\begin{array}{c}28.3 \\
( \pm 5.4)\end{array}$ & $\begin{array}{c}29.0 \\
( \pm 5.1)\end{array}$ & $\begin{array}{c}27.9 \\
( \pm 5.4) \\
\end{array}$ \\
\hline$<18.5 \mathrm{~kg} / \mathrm{m}^{2}$ & & $\begin{array}{c}15 \\
(0.7)\end{array}$ & & $\begin{array}{c}3 \\
(0.6)\end{array}$ & & $\begin{array}{c}3 \\
(0.41)\end{array}$ & $\begin{array}{c}5 \\
(2.5)\end{array}$ & $\begin{array}{c}5 \\
(0.6)\end{array}$ & $\begin{array}{c}2 \\
(1.0)\end{array}$ & $\begin{array}{c}12 \\
(0.9)\end{array}$ \\
\hline $18.5-24.9 \mathrm{~kg} / \mathrm{m}^{2}$ & & $\begin{array}{c}571 \\
(28.1)\end{array}$ & $\begin{array}{c}45 \\
(28.3)\end{array}$ & $\begin{array}{c}90 \\
(19.3)\end{array}$ & $\begin{array}{c}22 \\
(17.3)\end{array}$ & $\begin{array}{c}157 \\
(20.8)\end{array}$ & $\begin{array}{c}111 \\
(55.8)\end{array}$ & $\begin{array}{c}260 \\
(29.4)\end{array}$ & $\begin{array}{c}43 \\
(21.6)\end{array}$ & $\begin{array}{c}414 \\
(32.3)\end{array}$ \\
\hline $25-29.9 \mathrm{~kg} / \mathrm{m}^{2}$ & & $\begin{array}{c}817 \\
(40.1)\end{array}$ & $\begin{array}{c}72 \\
(45.3\end{array}$ & $\begin{array}{c}219 \\
(46.8)\end{array}$ & $\begin{array}{c}69 \\
(54.3)\end{array}$ & $\begin{array}{c}360 \\
(47.8)\end{array}$ & $\begin{array}{c}54 \\
(27.1) \\
\end{array}$ & $\begin{array}{c}332 \\
(37.6)\end{array}$ & $\begin{array}{c}71 \\
(35.7)\end{array}$ & $\begin{array}{c}457 \\
(35.7) \\
\end{array}$ \\
\hline$\geq 30 \mathrm{~kg} / \mathrm{m}^{2}$ & & $\begin{array}{c}632 \\
(31.1)\end{array}$ & $\begin{array}{c}42 \\
(26.4)\end{array}$ & $\begin{array}{c}156 \\
(33.3)\end{array}$ & $\begin{array}{c}36 \\
(28.4)\end{array}$ & $\begin{array}{c}234 \\
(31.0)\end{array}$ & $\begin{array}{c}29 \\
(14.6)\end{array}$ & $\begin{array}{c}286 \\
(32.4)\end{array}$ & $\begin{array}{c}83 \\
(41.7)\end{array}$ & $\begin{array}{c}398 \\
(31.1)\end{array}$ \\
\hline
\end{tabular}

to be 45-64-year age group, which was over three times higher than 30-44-year age group's (OR $=3.3712$; CI: 2.5901-4.3878). However, for people older than 54 years the risk is doubled (OR $=2.9605$; CI: 2.2418-3.9097).

The frequency of occurrence of diabetes, hypertension, coronary heart disease (CHD), circulatory deficiency or tuberculosis is significantly higher in rural than in urban areas (Table 2). It was not determined to be statistically significant in terms of other heart diseases, strokes, jaundice, cancer, chronic obstructive pulmonary disease (COPD), and asthma in urban and rural areas. Among chronic diseases in the investigated population, hypertension was the most common (36.7\%), followed by other heart disease $(12.1 \%)$, which was also noted to be more frequent among females than males (respectively, $14.1 \%$ vs. $6.8 \%)$, and then circulatory inefficiency (11.1\%), diabetes (7.3\%), liver diseases (6.5\%), which also appeared to be more frequent among females then males $(7.3 \%$ vs. $5.1 \%)$, and finally $\mathrm{CHD}$ (5.5\%) was significantly more frequent among males (7.3\% vs. 4.5$)$ (Table 2) [8-11].

\section{PROJECT COORDINATOR IN POLAND}

Research Coordinator: Prof. Witold Zatoński, Chair of Health Promotion Foundation.

\section{CO-COORDINATORS}

Prof. Andrzej Szuba, Wroclaw Medical University and dr hab. n. med. Katarzyna Zatońska, Department of Social Medicine, Wroclaw Medical University.

\section{DISCLOSURE}

Authors report no conflict of interest.

\section{References}

1. Zatoński WA, Zatoński M. Health in Polish People’s Republic. J Health Inequal 2016; 2: 7-16.

2. Teo K, Chow CK, Vaz M, et al. The Prospective Urban Rural Epidemiology (PURE) study: Examinig the impast of societal influences on chronic noncommunicable diseases in low-, middle-, and high-income countries. Am Heart J 2009; 158: 1-7.

3. Teo K, Lear S, Islam S, et al. Prevalence of a healthy lifestyle among individuals with cardiovascular disease in high-, middleand low-income countries: The Prospective Urban Rural Epidemiology (PURE) study. JAMA 2013; 309: 1613-1621.

4. Dehghan M, Ilow R, Zatonska K, et al. Development, reproducibility and validity of the food frequency questionnaire in the Poland arm of the Prospective Urban and Rural Epidemiological (PURE) study. J Hum Nutr Diet 2012; 25: 225-232. 


\begin{tabular}{|c|c|c|c|c|c|c|c|c|c|c|c|c|}
\hline & $\begin{array}{l}8 \\
\vdots \\
0\end{array}$ & $\begin{array}{l}\circ \\
\vdots \\
0\end{array}$ & $\begin{array}{l}\bar{\alpha} \\
\vdots \\
0\end{array}$ & 㝘 & 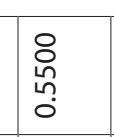 & $\mid \begin{array}{l}\text { to } \\
\text { m. } \\
0\end{array}$ & $\begin{array}{l}\frac{n}{0} \\
\frac{0}{0} \\
\end{array}$ & $\begin{array}{l}\text { 总 } \\
\text { o. } \\
\end{array}$ & $\frac{8}{\frac{8}{7}}$ & 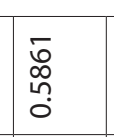 & $\begin{array}{l}\text { tे } \\
\text { oे } \\
0\end{array}$ & 亏े \\
\hline & \& $\stackrel{\text { F }}{\stackrel{F}{S}}$ & 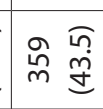 & 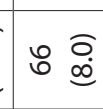 & $\stackrel{i}{i}$ & 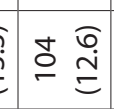 & 웜 & in & $\infty \underset{\mathbb{J}}{\mathbb{I}}$ & $\therefore \stackrel{\widehat{a}}{=}$ & m高 & $m \stackrel{\text { f }}{=}$ & 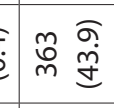 \\
\hline & 紸 & 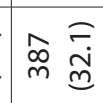 & 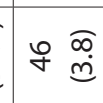 & 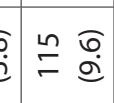 & 王 & $\simeq \stackrel{\text { nิ }}{=}$ & $\bar{\infty} \underset{\hat{\theta}}{\pi}$ & ᄀ $\overline{\mathscr{n}}$ & $\stackrel{\sim}{\sim} \overline{\mathrm{d}}$ & $g \stackrel{\widehat{q}}{\dot{q}}$ & $\simeq \stackrel{\sigma}{\stackrel{\sigma}{P}}$ & $\frac{9}{\partial \sigma}$ \\
\hline & ঃ & $\vdots$ & 产 & 产 & \begin{tabular}{|l|}
$\bar{g}$ \\
o. \\
\end{tabular} & 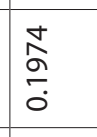 & \begin{tabular}{|l|} 
\\
ha. \\
o.
\end{tabular} & 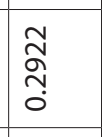 & $\begin{array}{l}\infty \\
\substack{0 \\
0 \\
0}\end{array}$ & $\begin{array}{l}\text { 㝵 } \\
\text { 品 }\end{array}$ & $\begin{array}{l}\infty \\
\vdots \\
0 \\
0\end{array}$ & 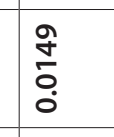 \\
\hline & $\therefore \stackrel{\widehat{m}}{E}$ & $\tilde{\approx} \widetilde{\sim}$ & 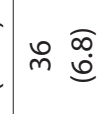 & 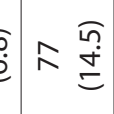 & 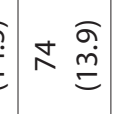 & 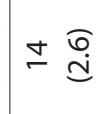 & $\stackrel{\rho}{m} \stackrel{\widehat{m}}{\Omega}$ & 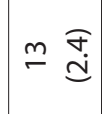 & 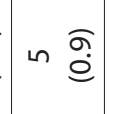 & ๗ & $\sim \stackrel{f}{\Theta}$ & $\bar{x} \overline{\widetilde{n}}$ \\
\hline & $\widetilde{m} \widehat{\underline{y}}$ & 宂 $\bar{్}$ & 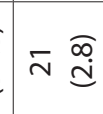 & 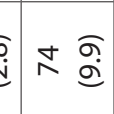 & 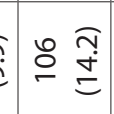 & $\simeq \stackrel{\sigma}{=}$ & in $\bar{x}$ & 요 & $\stackrel{i}{a}$ & 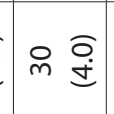 & $\pm \underset{\partial}{\sigma}$ & 合番 \\
\hline & $\overline{\bar{o}}$ & $\begin{array}{l}\tilde{\tilde{O}} \\
0 \\
0 \\
0\end{array}$ & $\begin{array}{l}\overline{\bar{g}} \\
\overline{0} \\
\end{array}$ & $\begin{array}{l}\tilde{z} \\
\tilde{N} \\
0 \\
0\end{array}$ & 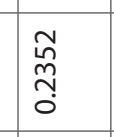 & 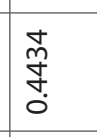 & 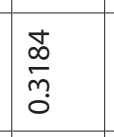 & $\begin{array}{l}\frac{8}{8} \\
\substack{0 \\
0}\end{array}$ & 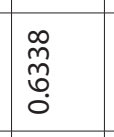 & 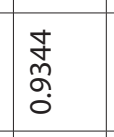 & 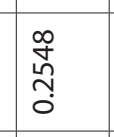 & 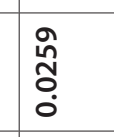 \\
\hline & 志 & $\approx \overline{\bar{g}}$ & 일 & 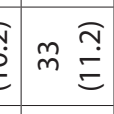 & 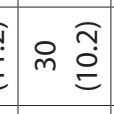 & $\circ \stackrel{\vec{g}}{\vec{g}}$ & $\simeq \overline{\overline{ \pm}}$ & 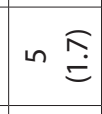 & 的 $\underset{E}{\mathbb{E}}$ & $\simeq \stackrel{\overline{ \pm}}{\bar{I}}$ & $-\widehat{\widehat{m}}$ & 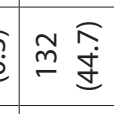 \\
\hline & ఇ $\widehat{\widehat{a}}$ & 必 & ๗ & $\bar{i} \bar{g}$ & $\stackrel{n}{m} \underset{\Sigma}{\mathbb{S}}$ & ○ & 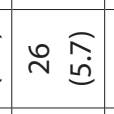 & 젰 & $\circ \widehat{\mathbb{d}}$ & $\stackrel{\infty}{-\infty}$ & n $\stackrel{\equiv}{=}$ & 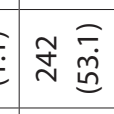 \\
\hline & 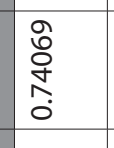 & $\begin{array}{l}\text { 惫 } \\
\text { on } \\
0\end{array}$ & $\begin{array}{l}\overline{\overline{0}} \\
\dot{0} \\
0\end{array}$ & $\begin{array}{l}\hat{o} \\
\stackrel{0}{0} \\
o \\
0\end{array}$ & $\begin{array}{l}0 \\
\grave{0} \\
0 \\
0\end{array}$ & $\begin{array}{l}\overline{\bar{\infty}} \\
\substack{\bar{\infty} \\
0 \\
0}\end{array}$ & 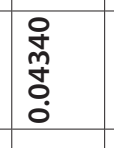 & 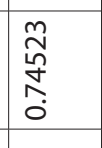 & 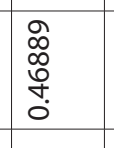 & $\begin{array}{l}\bar{\delta} \\
\vdots \\
\vdots \\
0 \\
0\end{array}$ & 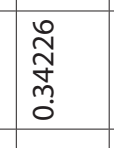 & $\begin{array}{l}\infty \\
\substack{\infty \\
\tilde{m} \\
0 \\
0}\end{array}$ \\
\hline & $\approx \stackrel{\widetilde{\Xi}}{\mathbb{S}}$ & 原离 & 的孚 & 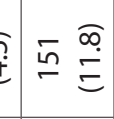 & 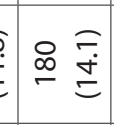 & $\stackrel{\sim}{\stackrel{\mathrm{d}}{\mathrm{d}}}$ & $\sin$ & òm $\widehat{\rho}$ & $\curvearrowright \stackrel{\widehat{\sigma}}{=}$ & in $\widehat{\widehat{q}}$ & 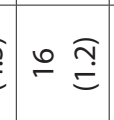 & 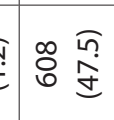 \\
\hline & in & 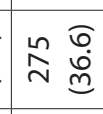 & 织 & I & $\because \begin{array}{c}\overline{0} \\
0\end{array}$ & $\simeq \stackrel{\sigma}{=}$ & $\stackrel{\infty}{\infty} \overline{\dot{\varphi}}$ & $\bar{\sim} \overline{\widetilde{d}}$ & $\bumpeq \bar{a}$ & 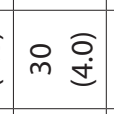 & $\circ \stackrel{\widehat{x}}{\dot{\infty}}$ & 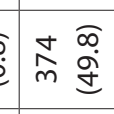 \\
\hline & 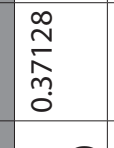 & $\begin{array}{l}\frac{\pi}{\pi} \\
\bar{\alpha} \\
0\end{array}$ & $\begin{array}{l}\bar{\alpha} \\
\tilde{\tilde{N}} \\
\tilde{\sigma} \\
\end{array}$ & $\begin{array}{l}\overline{\tilde{N}} \\
\tilde{N} \\
\tilde{D} \\
\bar{\Xi}\end{array}$ & $\begin{array}{l}\hat{\mathrm{O}} \\
\stackrel{0}{0} \\
0 \\
0\end{array}$ & 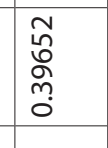 & 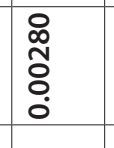 & 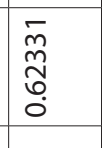 & 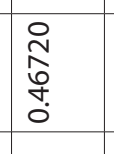 & $\begin{array}{l} \\
f \\
0 \\
0 \\
0\end{array}$ & 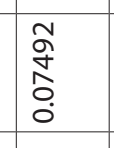 & 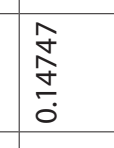 \\
\hline & $\approx \stackrel{\bar{\sigma}}{\stackrel{\rho}{=}}$ & $\pm \widetilde{\widetilde{M}}$ & ๗ & 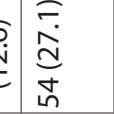 & \& $\overline{\mathscr{d}}$ & $\pm \stackrel{\Xi}{S}$ & $\sim \stackrel{\sigma}{\Xi}$ & $\circ \overline{\dot{m}}$ & in $\overline{\mathrm{g}}$ & $\circ \stackrel{\widehat{c}}{\hat{\omega}}$ & $\sim \stackrel{\widehat{Q}}{\dot{Q}}$ & $\Varangle \stackrel{\square}{d}$ \\
\hline : & $m \stackrel{\widetilde{\Omega}}{\stackrel{S}{g}}$ & $\approx \widetilde{0}$ & $\approx \stackrel{\widehat{N}}{\underline{N}}$ & $\approx \sim \underset{\widetilde{n}}{\underline{d}}$ & 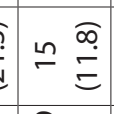 & $\circ \stackrel{\widetilde{I}}{\mathbb{1}}$ & $m \underset{\mathcal{I}}{\mp}$ & $\infty \widehat{\widehat{m}}$ & in $\bar{x}$ & in $\bar{x}$ & nn & 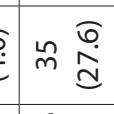 \\
\hline & 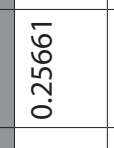 & $\begin{array}{l}n \\
0 \\
0 \\
0 \\
0\end{array}$ & 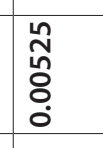 & $\begin{array}{l} \\
\\
0.0 \\
0 \\
0 \\
0\end{array}$ & $\begin{array}{l}\hat{a} \\
\hat{\vdots} \\
0 \\
0\end{array}$ & 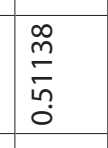 & 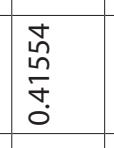 & 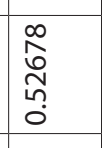 & 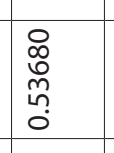 & \begin{tabular}{l}
\multirow{̃}{\Xi}{} \\
o. \\
0
\end{tabular} & $\begin{array}{l}\overline{\mathrm{o}} \\
\stackrel{0}{0} \\
0 \\
0\end{array}$ & 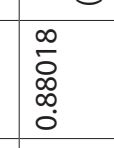 \\
\hline & 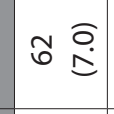 & হ্ল & $\tilde{m}$ & 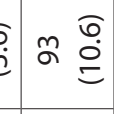 & 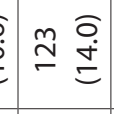 & $=\stackrel{\widetilde{\Im}}{\Xi}$ & $0 \stackrel{\sqrt[a]{\Sigma}}{\Sigma}$ & $\underset{\sim}{\infty} \underset{\tilde{c}}{\widehat{\widetilde{c}}}$ & $=\stackrel{m}{=}$ & 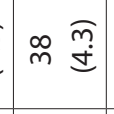 & 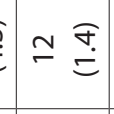 & 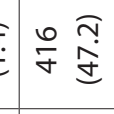 \\
\hline & $F_{\infty}^{\infty} \underset{\infty}{\infty}$ & 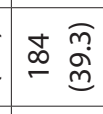 & $m$ & $g$ g & $\approx \widehat{g}$ & $+\widehat{\widehat{g}}$ & $\stackrel{\infty}{\sim} \underset{\dot{\rho}}{\widehat{\sigma}}$ & 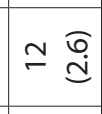 & $\sigma \stackrel{\widehat{\sigma}}{=}$ & $\stackrel{\infty}{\infty}$ & $-\widehat{\widehat{s}}$ & 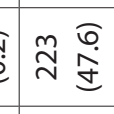 \\
\hline & $\begin{array}{l}\text { 总 } \\
\text { م. } \\
\end{array}$ & 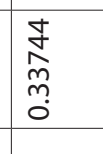 & , & 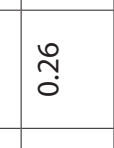 & 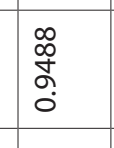 & 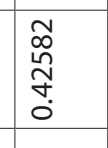 & 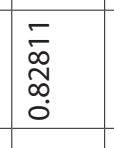 & 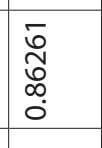 & $\stackrel{\circ}{\circ}$ & 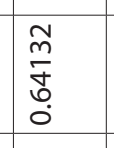 & $\begin{array}{l}\tilde{N} \\
\tilde{N} \\
\sigma \\
0\end{array}$ & 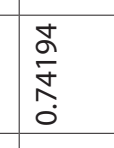 \\
\hline 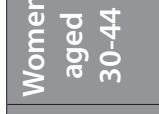 & $m \stackrel{\sqrt[n]{g}}{=}$ & 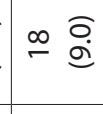 & 0 & $+\stackrel{d}{\mathbb{~}}$ & $\stackrel{0}{a}$ & $-\sqrt{\frac{\pi}{9}}$ & $\infty \stackrel{\widehat{o}}{\dot{\Phi}}$ & $-\stackrel{\sqrt{n}}{\stackrel{n}{9}}$ & $\sim \stackrel{\widehat{O}}{\dot{S}}$ & $\wedge \stackrel{\sqrt[n]{n}}{n}$ & $\sim \stackrel{\widehat{\rho}}{=}$ & 도 \\
\hline${ }_{0}^{\circ} \%$ & $m \stackrel{\sigma}{\Theta}$ & $\propto \stackrel{\widetilde{I}}{\mathbb{I}}$ & 0 & $-\overline{\grave{g}}$ & $\infty \underset{\hat{n}}{\sigma}$ & $\sim \widehat{\widetilde{g}}$ & $\wedge \overline{\mathscr{g}}$ & $-\stackrel{\bar{o}}{\stackrel{9}{9}}$ & $-\stackrel{\bar{o}}{\stackrel{S}{S}}$ & 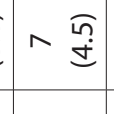 & 0 & 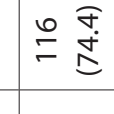 \\
\hline & g. & 里总 & $\cong \sqrt{n}$ & 该 & 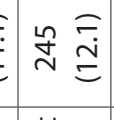 & $\stackrel{\infty}{\infty} \underset{\rho}{\stackrel{a}{E}}$ & 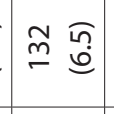 & \& $\overline{\stackrel{m}{m}}$ & $\stackrel{i}{\pi} \underset{\Xi}{\pi}$ & $\infty \underset{\mathcal{g}}{\widehat{g}}$ & $\approx$ & 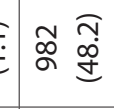 \\
\hline & 苋 & 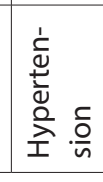 & & & & & & 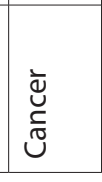 & 을 & 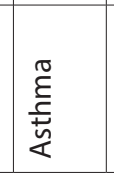 & & 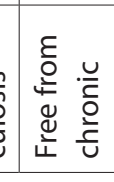 \\
\hline
\end{tabular}


5. Rosengren A, Teo K, Rangarajan S, et al. Psychosocial factors and obesity in 17 high-, middle- and low-income countries: the Prospective Urban Rural Epidemiologic study. Int J Obes 2015; 39: 1217-1223.

6. Leong DP, Teo KK, Rangarajan S, et al. Prognostic value of grip strength: findings from the Prospective Urban Rural Epidemiology (PURE) study. Lancet 2015; 386: 266-273.

7. Dagenais GR, Gerstein HC, Zhang X, et al. Variations in diabetes prevalence in low-, middle-, and high-income countries: results from the Prospective Urban and Rural Epidemiology Study. Diabetes Care 2016; 39: 780-787.

8. Yusuf S, Islam S, Chow CK, et al., on behalf of the Prospective Urban Rural Epidemiology (PURE). Use of secondary prevention drugs for cardiovascular disease in the community in high-income, middle-income, and low-income countries (the PURE Study): a prospective epidemiological survey. Lancet 2011; 378: 1231-1243.

9. Savell E, Gilmore AB, Sims M, et al. The environmental profile of a community's health: a cross-sectional study on tobacco marketing in 16 countries. Bull World Health Organ 2015; 93: 851-861.

10. Smyth A, Teo KK, Rangarajan S, et al.; the PURE Investigators. Alcohol consumption and cardiovascular disease, cancer, injury, admission to hospital, and mortality: a prospective cohort study. Lancet 2015; 386: 1945-1954.

11. Miller V, Yusuf S, Chow CK, et al. Availability, affordability, and consumption of fruits and vegetables in 18 countries across income levels: findings from the Prospective Urban Rural Epidemiology (PURE) study. Lancet Glob Health 2016; 4: e695-e703.

\section{AUTHORS' CONTRIBUTIONS}

$K Z$, WAZ, AS prepared concept and design of the publication. KZ collected and analysed data. KZ and WAZ wrote the article. WAZ and AS participated in its critical revision and final approval. 